\title{
METODOLOGIAS PARA ESTIMATIVA DOS PARÂMETROS DA EQUAÇÃO DE CHUVAS INTENSAS NO ESTADO DO ESPÍRITO SANTO
}

Rafael Salgado de Senna ${ }^{1}$, Alexandre Cândido Xavier², Julião Soares de Sousa Lima ${ }^{3}$, Roberto Avelino Cecílio ${ }^{4}$

RESUMO

Este trabalho foi realizado com o objetivo de avaliar a interpolação dos parâmetros da equação de chuvas intensas, pelo método inverso de uma potência da distância, e comparar com o método vizinho mais próximo, para todo o Estado do Espírito Santo. Foi realizada a interpolação dos parâmetros "K", "a", "b" e "c" da equação de chuvas intensas, utilizandose o interpolador "Inverso de uma potência da distância", com cinco diferentes potências, a saber: potências 1, 2, 3, 4 e 5 e "Média aritmética simples" das três vizinhas mais próximas de cada estação. Foram consideradas, para cálculo da intensidade máxima, a média de precipitação $\left(\mathrm{i}_{\mathrm{m}}\right)$, todas as combinações possíveis entre os períodos de retorno $(\mathrm{T})$ iguais a 2, 5, 10, 20, 50, e 100 anos e as durações da precipitação (t) iguais a 10, 20, 30, 40, 50, 60, 120, 240, 360, 720 e 1440 minutos, totalizando 66 combinações para cada uma das 20 estações pluviométricas. De posse dos parâmetros interpolados de cada uma das estações, foram calculados os valores de $\mathrm{i}_{\mathrm{m}}$, por meio da equação de chuvas intensas. Utilizando-se os valores de $\mathrm{i}_{\mathrm{m}}$ calculados e interpolados, foi calculado o erro médio percentual. Para finalizar, procedeu-se a uma análise de regressão dos dados, correlacionando-se o erro médio percentual com as diferentes potências. O método vizinho mais próximo proporcionou um erro médio de 7,03\%, enquanto que o método do inverso de uma potência da distância apresentou um erro médio de 16,4\%. Assim, o método vizinho mais próximo teve melhores resultados para a interpolação dos parâmetros da equação de chuvas intensas para o Estado do Espírito Santo.

Palavras-chave: precipitação pluviomêtrica, modelagem hidrológica, escoamento superficial

\section{INTERPOLATION METHODS OF THE PARAMETERS OF THE INTENSE RAIN EQUATION IN ESPIRITO SANTO STATE}

\begin{abstract}
This work has the objective of evaluating the interpolation of the parameters of the intense rain equation, by the inverse method of one potency of the distance, using five different potencies and comparing all with the close neighbors method of the Espírito Santo state. The interpolation of parameters "K", "a", "b" and "c" of the equation for intense rain was carried through, using the inverse interpolator of one potency of the distance with five different potencies, being: potency 1, 2, 3, 4 and 5 and the close neighbors interpolator. Considering all the possible combinations between the periods of return (T) equals 2, 5, 10, 20, 50, and 100 years and the precipitation duration (t) equals 10, 20, 30, 40, 50, 60, 120, 240, 360,720 and 1440 minutes, totalizing 66 combinations for each one of the 20 stations. In ownership of the parameters interpolated of each one of the stations, the values of the average maximum precipitation intensity had been calculated $\left(i_{m}\right)$, by means of the intense rain equation. Using the calculated values of $i_{m}$ and the interpolated ones, the percentage of average error. To finish, an analyzes of regression of the data was proceeded, correlating the average error percentage with the different potencies. The closest neighbor method provided an average error of $7,03 \%$ and for the method of the inverse of a potency of the distance a value of $16,4 \%$ of average error. Being thus, the closest neighbor method had better resulted for the interpolation of the parameters of the intense rain equation specifically for the state of Espirito Santo.
\end{abstract}

Keywords: precipitation hydrologic modeling superficial draining

\section{Recebido para publicação em 30/03/2009. Aprovado em 08/04/2010}

1- Mestrando, UFES-DER, Alegre ES, rafassenna@yahoo.com.br,

2- D.S Eng. Rural, Prof. Adjunto, DER, UFES, Alegre ES, xavier@cca.ufes.br,

3- D.S Eng. Rural, Prof. Adjunto, DER, UFES, Alegre ES, juliaosslima@cca.ufes.br,

4- D.S. Eng. Florestal, Prof. Adjunto, Bolsista de Produtividade em Pesquisa do CNPq, DEF, UFES, Alegre ES, racecilio@yahho.com.br, 


\section{INTRODUÇÃO}

Chuvas intensas, também denominadas chuvas extremas ou máximas, são aquelas que apresentam grande lâmina precipitada, durante pequeno intervalo de tempo (SILVA et al., 2003). Uma das características dessas chuvas é possuírem distribuição irregular, tanto espacial, quanto temporalmente (ARAÚJO et al., 2008).

Os estudos de chuvas intensas são especialmente importantes, por sua relação com a ocorrência e a magnitude do escoamento superficial, que pode ocasionar severos prejuízos em áreas agrícolas e urbanas, tais como a destruição de barragens, a erosão do solo, o assoreamento de corpos hídricos, os alagamentos em áreas urbanizadas e lavouras, dentre outros (SILVA et al., 2003). Portanto, a determinação da intensidade das precipitações intensas é importante para o dimensionamento de estruturas destinadas ao correto manejo de bacias hidrográficas, ao controle e proteção contra inundações e às práticas de conservação do solo e de produção de água. A estimativa mais precisa possível de uma chuva intensa é extremamente importante para que um projeto seja viável, tanto do ponto de vista técnico quanto econômico (MELLO et al., 2003).

A determinação das equações de chuvas intensas apresenta grandes dificuldades, em função da escassez de registros pluviográficos, da baixa densidade da rede pluviográfica e do pequeno período de observações disponível. Além disso, a metodologia para sua obtenção exige um exaustivo trabalho de tabulação, análise e interpretação de grande quantidade de pluviogramas (OLIVEIRA et al., 2000), o que faz com que haja indisponibilidade desta informação para a grande maioria das localidades brasileiras, em especial do Espírito Santo. Uma alternativa comumente utilizada para contornar esse problema tem sido a utilização dos parâmetros "K", "a", "b" e "c", obtidos em postos meteorológicos mais próximos da localidade na qual um projeto qualquer é realizado. Contudo, esse procedimento pode levar a estimativas não confiáveis (SILVA et al., 1999).

Trabalhos (PRUSKI et al., 1997; SILVA et al., 1999) sugerem a utilização do método de interpolação espacial "Inverso do quadrado da distância" para a determinação dos parâmetros da equação de chuvas intensas para qualquer localidade. Baseando-se no trabalho de Cecílio \& Pruski et al. (2003), realizado para Minas Gerais, Pruski et al. (2006) sugerem a utilização do interpolador espacial "inverso da quinta potência da distância" para espacialização dos parâmetros da equação de chuvas intensas no Brasil.

Em razão do exposto, o objetivo do presente trabalho foi avaliar, para o estado do Espírito Santo, diferentes métodos de interpolação dos parâmetros da equação de chuvas intensas, sendo avaliados o método "Inverso de uma potência da distância", utilizando-se cinco diferentes potências, e a "Média aritmética simples", considerando-se as três estações mais próximas da localidade de interesse.

\section{MATERIAL E MÉTODOS}

Mapa do Estado do Espírito Santo (Figura 1), indicando a localização pontual das 20 estações utilizadas como teste no presente estudo.

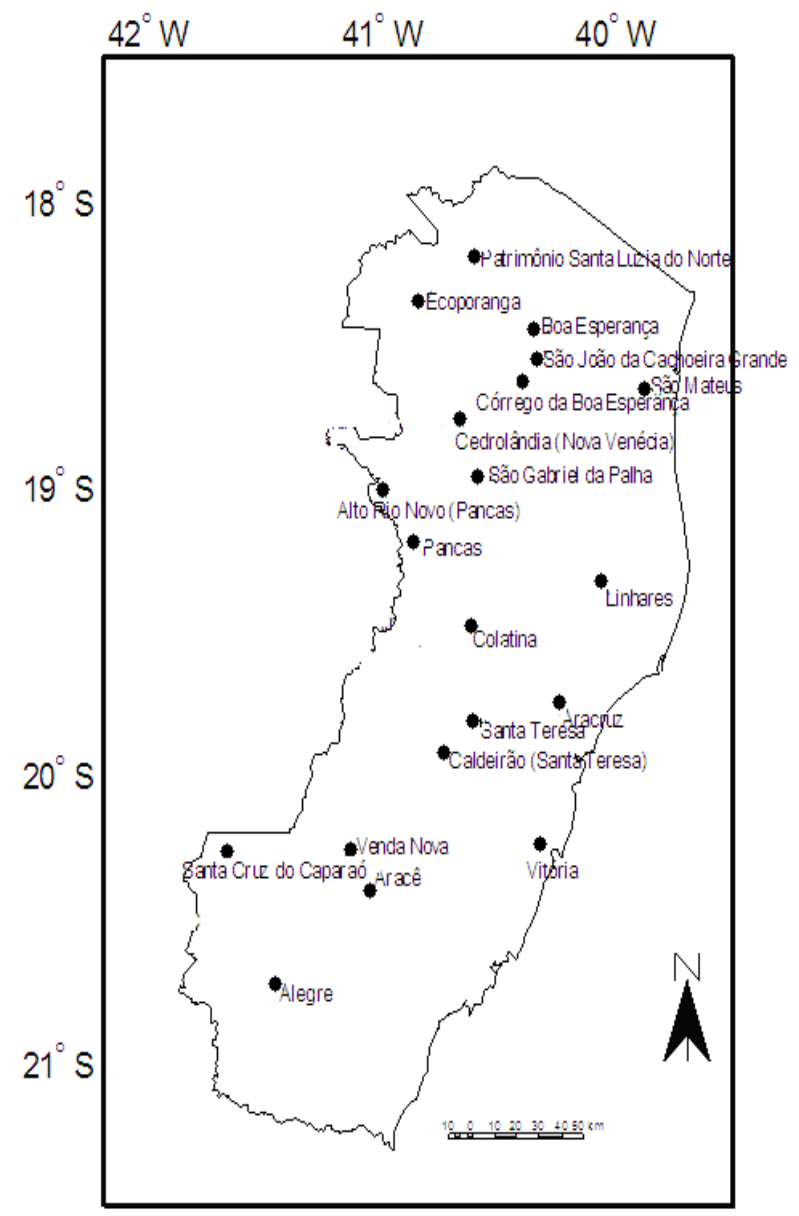

Figura 1. Mapa do Estado do Espírito Santo com a localização das estações utilizadas. 
Quadro 1. Estações utilizadas para teste com as respectivas coordenadas geográficas e parâmetros da equação de chuças intensas

\begin{tabular}{|c|c|c|c|c|c|c|}
\hline Estação & Latitude & Longitude & $\mathbf{K}$ & $\mathbf{a}$ & b & c \\
\hline Alegre & $20^{\circ} 46^{\prime} 00^{\prime \prime}$ & $41^{\circ} 28^{\prime} 00^{\prime \prime}$ & 1497,781 & 0,258 & 19,294 & 0,855 \\
\hline Alto Rio Novo (Pancas) & $19^{\circ} 03 ’ 33^{\prime \prime}$ & $41^{\circ} 01 ’ 39^{\prime \prime}$ & 1908,138 & 0,208 & 18,432 & 0,926 \\
\hline Aracê (Domingos Martins) & $20^{\circ} 22^{\prime} 00^{\prime \prime}$ & $41^{\circ} 03^{\prime} 40^{\prime \prime}$ & 880,474 & 0,175 & 9,722 & 0,729 \\
\hline Aracruz & $19^{\circ} 49^{\prime} 00^{\prime \prime}$ & $40^{\circ} 15^{\prime} 00^{\prime \prime}$ & 1298,382 & 0,200 & 20,981 & 0,786 \\
\hline Boa Esperança & $18^{\circ} 31^{\prime} 00^{\prime \prime}$ & $40^{\circ} 19^{\prime} 00^{\prime \prime}$ & 596,380 & 0,230 & 8,534 & 0,670 \\
\hline Caldeirão (Santa Teresa) & $19^{\circ} 57^{\prime} 18^{\prime \prime}$ & $40^{\circ} 44^{\prime} 30^{\prime \prime}$ & 3777,338 & 0,196 & 46,751 & 0,947 \\
\hline Cedrolândia (Nova Venécia) & $18^{\circ} 48^{\prime} 53^{\prime \prime}$ & $40^{\circ} 41^{\prime} 27^{\prime \prime}$ & 4000,000 & 0,178 & 51,492 & 0,920 \\
\hline Colatina & $19^{\circ} 32^{\prime} 00^{\prime \prime}$ & $40^{\circ} 38^{\prime} 45^{\prime \prime}$ & 709,931 & 0,201 & 7,331 & 0,687 \\
\hline $\begin{array}{l}\text { Córrego da Boa Esperança } \\
\text { (Nova Venécia) }\end{array}$ & $18^{\circ} 42^{\prime} 00^{\prime \prime}$ & $40^{\circ} 26^{\prime} 30^{\prime \prime}$ & 4350,786 & 0,202 & 40,254 & 1,003 \\
\hline Ecoporanga & $18^{\circ} 21^{\prime} 57^{\prime \prime}$ & $40^{\circ} 50^{\prime} 26^{\prime \prime}$ & 5666,170 & 0,197 & 43,631 & 1,055 \\
\hline Linhares & $19^{\circ} 24^{\prime} 00^{\prime \prime}$ & $40^{\circ} 04^{\prime} 00^{\prime \prime}$ & 3647,235 & 0,223 & 20,665 & 1,000 \\
\hline Pancas & $19^{\circ} 13^{\prime} 13^{\prime \prime}$ & $40^{\circ} 51^{\prime} 12^{\prime \prime}$ & 1227,888 & 0,185 & 20,628 & 0,758 \\
\hline $\begin{array}{l}\text { Patrimônio Santa Luzia do } \\
\text { Norte (Ecoporanga) }\end{array}$ & $18^{\circ} 12^{\prime} 21^{\prime \prime}$ & $40^{\circ} 36^{\prime} 15^{\prime \prime}$ & 2407,091 & 0,187 & 34,383 & 0,877 \\
\hline Santa Cruz do Caparaó (Iúna) & $20^{\circ} 19^{\prime} 22^{\prime \prime}$ & $41^{\circ} 42^{\prime} 15^{\prime \prime}$ & 3873,672 & 0,180 & 35,418 & 0,986 \\
\hline Santa Teresa & $19^{\circ} 51^{\prime} 00^{\prime \prime}$ & $40^{\circ} 36^{\prime} 00^{\prime \prime}$ & 632,265 & 0,247 & 13,543 & 0,714 \\
\hline São Gabriel da Palha & $19^{\circ} 01^{\prime} 00^{\prime \prime}$ & $40^{\circ} 34^{\prime} 00^{\prime \prime}$ & 1309,205 & 0,230 & 15,375 & 0,821 \\
\hline $\begin{array}{c}\text { São João da Cachoeira Grande } \\
\text { (São Mateus) }\end{array}$ & $18^{\circ} 33^{\prime} 50^{\prime \prime}$ & $40^{\circ} 20^{\prime} 10^{\prime \prime}$ & 5829,147 & 0,192 & 33,421 & 1,089 \\
\hline São Mateus & $18^{\circ} 42^{\prime} 00^{\prime \prime}$ & $39^{\circ} 51^{\prime} 00^{\prime \prime}$ & 4999,380 & 0,191 & 49,999 & 0,983 \\
\hline Venda Nova & $20^{\circ} 19^{\prime} 00^{\prime \prime}$ & $41^{\circ} 10^{\prime} 00^{\prime \prime}$ & 4147,062 & 0,205 & 33,842 & 1,000 \\
\hline Vitória & $20^{\circ} 19^{\prime} 00^{\prime \prime}$ & $40^{\circ} 20^{\prime} 00^{\prime \prime}$ & 4003,611 & 0,203 & 49,997 & 0,931 \\
\hline Média & & & 2838,097 & 0,204 & 28,685 & 0,887 \\
\hline
\end{tabular}

Foram utilizados os valores dos parâmetros "K", "a", "b" e "c" referentes às 20 estações localizadas no Espírito Santo, determinados por Freitas et al. (2001). Para efeito de borda, utilizaram-se os valores de "K", "a", "b" e "c" de mais 146 estações distribuídas nos estados da Bahia, Rio de Janeiro e Minas Gerais, determinados por Freitas et al. (2001), Pinto (1999), Silva et al. (2002), totalizando-se, assim, 166 estações utilizadas no presente estudo.

De acordo com Villela \& Mattos (1975), as chuvas intensas podem ser caracterizadas por meio da equação de intensidade-duração-frequência da precipitação, ou, simplesmente, equação de chuvas intensas (equação 1).

$$
i_{m}=\frac{K T^{a}}{(t+b)^{c}}
$$

em que,

$\mathrm{i}_{\mathrm{m}}=$ intensidade máxima média de precipitação $(\mathrm{mm} / \mathrm{h})$;

$\mathrm{T}=$ período de retorno(anos);

$\mathrm{t}=$ duração da precipitação (minutos);

$\mathrm{K}, \mathrm{a}, \mathrm{b}, \mathrm{c}=$ parâmetros de ajuste estatístico.

Referentes a localização de cada estação. Foram utilizados dois métodos de interpolação espacial para estimação dos parâmetros "K", "a", "b" e "c" da equação de chuvas intensas:

i) o interpolador "Inverso de uma Potência da Distância" (IPD) (equação 2), considerando-se cinco diferentes potências (1, 2, 3, 4 e 5);

ii) a "Média aritmética simples", que consiste na média aritmética dos valores dos parâmetros das três estações mais próximas da localidade de interesse. 


$$
G_{i}=\frac{\sum_{i=1}^{n}\left(\frac{x_{i}}{d_{i}^{m}}\right)}{\sum_{i=1}^{n}\left(\frac{1}{d_{i}^{m}}\right)}
$$

em que,

$G_{i}=$ valor estimado na posição interpolada;

$x_{i}=$ valor do i-ésimo posto de controle;

$d_{i}=$ distância euclidiana entre o i-ésimo posto de controle e a posição interpolada;

$m=$ potência da distância euclidiana;

$n=$ número de vizinhos considerados.

A análise dos métodos de interpolação foi feita com a utilização do método de validação cruzada, descrito por Myers (1997) e adaptado por Cecílio \& Pruski (2003) e por Amorim et al. (2008). Esse método consistiu na extração individual, da base de dados das 166 estações, de cada uma das 20 estações do Espírito Santo, com posterior realização das interpolações, por cada um dos métodos, de cada um dos quatro parâmetros da equação de chuvas intensas. Após a realização da interpolação, a estação extraída era recolocada na base de dados e outra era escolhida para a extração. Desta forma, para cada uma das 20 estações, foi possível comparar os valores reais e estimados dos quatro parâmetros da equação de chuvas intensas.

Conforme idealizado por Cecílio \& Pruski (2003), a avaliação do IPD foi feita considerandose todas as possíveis combinações entre os parâmetros interpolados, considerando-se cada uma das cinco potências, totalizando, assim, 625 combinações.

Os valores dos parâmetros "K", "a", "b" e "c" estimados por cada método avaliado foram armazenados a fim de que, posteriormente, e para cada uma das 20 estações, fosse procedido o cálculo da intensidade de precipitação $\left(\mathrm{i}_{\mathrm{m} \text { (int) }}\right)$ pela equação 1. Para tanto, consideraram-se períodos de retorno (T) iguais a 2, 5, 10, 20, 50 e 100 anos e durações da precipitação $(\mathrm{t})$ iguais a 10, 20, 30, $40,50,60,120,240,360,720$ e 1440 minutos, resultando em 66 combinações diferentes. Os valores de $\mathrm{i}_{m(\text { int })}$ foram então comparados com aqueles calculados pelos parâmetros originais de cada estação $\left(\mathrm{i}_{m(c a l)}\right)$, segundo a equação:

$$
E=\frac{100\left|i_{m(\text { calc. })}-i_{m(\text { int. })}\right|}{i_{m(\text { calc. })}}
$$

em que,

\section{$E \quad$ é o erro percentual da estimativa.}

$\mathrm{O} E P$ foi calculado para as intensidades máximas médias de precipitação, oriundas de todas as combinações de $\mathrm{t}$ e $\mathrm{T}$ de todas as estações, sendo, por fim, calculada a média destes EP's (erro médio - EMP) para cada uma das 625 combinações das potências para estimativa dos parâmetros pelo método IPD.

Para avaliar a influência das diferentes potências no IPD, utilizou-se análise de regressão entre o erro médio e as cinco diferentes potências para cada parâmetro. Foram avaliados na regressão o nível descritivo de significância (valor-p) e o coeficiente de determinação $\left(\mathrm{R}^{2}\right)$.

\section{RESULTADOS E DISCUSSÃO}

\section{Avaliação do método inverso de uma potência da distância (IPD)}

No Quadro 2, estão apresentadas as combinações entre potências do IPD, cuja interpolação dos parâmetros "K", "a", "b" e "c" melhor estimou, em média, os valores de $i_{m}$ para o Espírito Santo, considerando-se todas as durações de precipitação e períodos de retorno avaliados no presente trabalho. Também são apresentados os erros médios percentuais, propiciados por cada combinação.

Pode-se verificar que para 20 das 625 combinações realizadas, o erro médio percentual (EMP) foi pequeno, com valores variando entre 16,4 e 17,9\% (Quadro 2). No geral, as potências do IPD que propiciaram menores erros médios foram as maiores potências para o parâmetro K; as menores potências para o parâmetro a; e as potências superiores a três para os parâmetros b e c.

Observa-se que, das 625 combinações avaliadas, aquela que apresentou o menor EMP $(16,4 \%)$ foi a utilização do inverso da quinta potência da distância para a interpolação dos parâmetros " $\mathrm{K}$ " e "b", o inverso da potência unitária da distância para "a" e o inverso da quarta potência da distância para o parâmetro "c". 
Quadro 2. Combinações entre as potências avaliadas e respectivos erros médios percentuais

\begin{tabular}{|c|c|c|c|c|}
\hline \multicolumn{4}{|c|}{ Potência utilizada na interpolação pelo IPD } & \multirow{2}{*}{$\begin{array}{c}\text { Erro Médio Percentual } \\
(\%)\end{array}$} \\
\hline $\mathbf{K}$ & $\mathbf{a}$ & b & c & \\
\hline 5 & 1 & 5 & 4 & 16,4 \\
\hline 5 & 1 & 4 & 4 & 16,5 \\
\hline 5 & 1 & 3 & 4 & 16,9 \\
\hline 5 & 2 & 5 & 4 & 17,1 \\
\hline 5 & 2 & 4 & 4 & 17,2 \\
\hline 5 & 1 & 5 & 3 & 17,3 \\
\hline 4 & 1 & 5 & 3 & 17,3 \\
\hline 5 & 3 & 5 & 4 & 17,3 \\
\hline 4 & 1 & 4 & 3 & 17,3 \\
\hline 5 & 4 & 5 & 4 & 17,4 \\
\hline 5 & 5 & 5 & 4 & 17,4 \\
\hline 5 & 3 & 4 & 4 & 17,5 \\
\hline 5 & 5 & 4 & 4 & 17,5 \\
\hline 5 & 4 & 4 & 4 & 17,5 \\
\hline 5 & 1 & 4 & 5 & 17,6 \\
\hline 5 & 1 & 5 & 5 & 17,6 \\
\hline 5 & 1 & 3 & 5 & 17,6 \\
\hline 5 & 1 & 4 & 3 & 17,7 \\
\hline 5 & 2 & 3 & 4 & 17,8 \\
\hline 4 & 1 & 3 & 3 & 17,9 \\
\hline
\end{tabular}

Trabalho semelhante, realizado por Cecílio \& Pruski (2003) para o Estado de Minas Gerais, em que os autores utilizaram 171 estações, mostrou que 28 das 625 combinações avaliadas apresentaram EMP variando entre 18,6 e 19,8\% (valores estatisticamente não diferentes). Estes valores de EMP têm magnitude semelhante aos encontrados no presente trabalho, embora sejam um pouco menores.

Cecílio \& Pruski (2003) encontraram que a interpolação do IPD, feita utilizando-se o inverso da quinta potência da distância para " $\mathrm{K}$ " e "c", o inverso da distância para "a" e o inverso do cubo da distância para "b" apresentou o menor EMP (18,6\%). Esta mesma combinação encontra-se entre as 20 de melhor desempenho no presente trabalho, todavia, com menor EMP $(17,6 \%)$.

Convém ressaltar que Cecílio \& Pruski (2003) avaliaram os valores de $i_{m}$ apenas para período de retorno de 10 anos e para a duração da precipitação de $60 \mathrm{~min}$. Salienta-se que, no presente trabalho, por ter-se avaliado 66 combinações entre o período de retorno $(\mathrm{T})$ e a duração da precipitação (t), os resultados mostrados podem ser considerados mais robustos.

Na Figura 2, estão apresentadas as relações entre o erro médio percentual de $\mathrm{i}_{\mathrm{m}}$ das 20 estações do ES e as cinco diferentes potências do IPD utilizadas para estimar os parâmetros
"K", "a", "b" e "c". Para cada potência, estão relacionadas 125 combinações que existem entre ela e as potências dos outros parâmetros, totalizando 625 combinações para cada parâmetro.

Somente os parâmetros: "K" e "c" apresentaram correlação (valor-p $<0,05)$ entre o erro médio e as diferentes potências, com um $\mathrm{R}^{2}$ igual a 0,48 e 0,05 , respectivamente. Sendo assim, ao interpolar esses parâmetros, recomenda-se a utilização das maiores potências para estimar o parâmetro " $\mathrm{K}$ " e das menores potências para estimar o parâmetro "c" do IPD.

\section{Avaliação da média das três estações mais próximas}

No Quadro 3, estão apresentados, para cada estação do Espírito Santo avaliada no presente trabalho, os erros médios percentuais fornecidos pelo método da "Média aritmética simples" entre as três estações mais próximas, na estimativa de $i_{m}$, considerando-se todos os períodos de retorno e as durações de precipitação avaliadas. O EMP de cada estação variou de 0,6 a 20,6\% em São Gabriel da Palha e Santa Teresa, respectivamente. O erro médio geral, considerando-se as 20 estações, foi de $7,0 \%$, valor muito inferior àquele obtido pela melhor combinação entre potências do método IPD $(16,4 \%)$. 

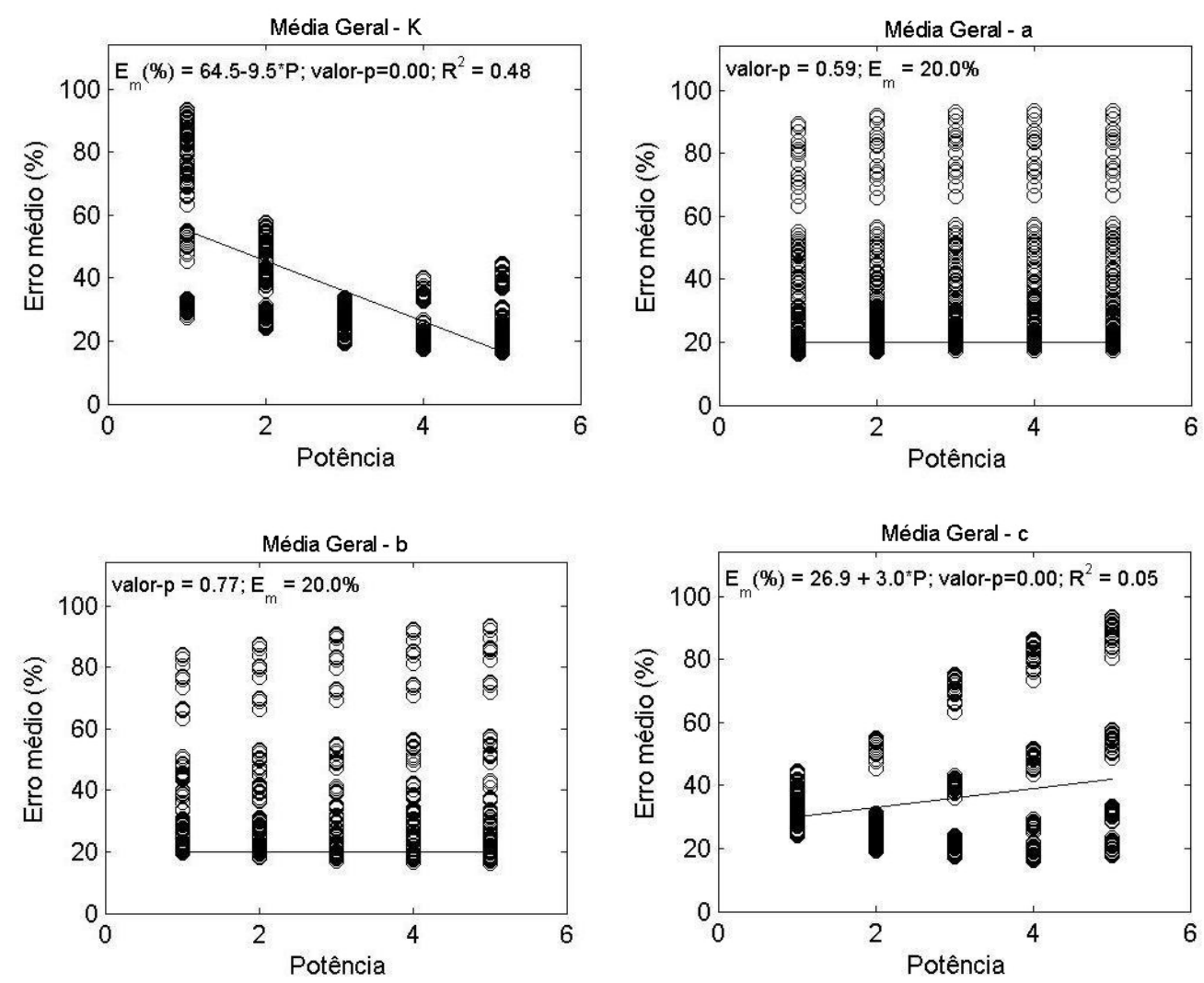

Figura 2. Análise de regressão dos parâmetros K, a, b e c referente à média geral das 20 estações

Quadro 3. Erros médios percentuais, obtidos com a aplicação do método da "Média aritmética simples" das três estações mais próximas

\begin{tabular}{cc}
\hline Estação & Erro médio percentual (\%) \\
Alegre & 3,4 \\
Ecoporanga & 2,5 \\
Linhares & 17,7 \\
Pancas & 1,2 \\
Santa Teresa & 20,6 \\
Venda Nova & 4,1 \\
Alto Rio Novo (Pancas) & 10,7 \\
Aracê (Domingos Martins) & 11,4 \\
Aracruz & 3,3 \\
Boa Esperança & 1,3 \\
Caldeirão (Santa Teresa) & 4,7 \\
Cedrolândia (Nova Venécia) & 9,0 \\
Colatina & 14,2 \\
Córrego da Boa Esperança & 5,4 \\
Patrimônio Santa Luzia do Norte (Ecoporanga) & 0,9 \\
Santa Cruz do Caparaó (Iúna) & 6,6 \\
São Gabriel da Palha & 0,6 \\
São João da Cachoeira Grande & 2,9 \\
São Mateus & 8,5 \\
Vitória & 11,4 \\
Média geral das 20 estações & $\mathbf{7 , 0}$ \\
\hline
\end{tabular}




\section{CONCLUSÕES}

- A interpolação dos parâmetros da equação de chuvas intensas para o estado do Espírito Santo, utilizando-se o método do inverso de uma potência da distância, propicia melhores estimativas da intensidade máxima média de precipitação, quando são utilizadas menores potências para o parâmetro "a" e maiores potências para os parâmetros "K", "b" e "c".

- O método da média aritmética simples entre as três estações mais próximas, aplicado na estimativa dos parâmetros da equação de chuvas intensas para o Estado do Espírito Santo, apresentou melhor desempenho das intensidades máximas médias de precipitação que o método do inverso de uma potência da distância.

\section{AGRADECIMENTOS}

Os autores agradece a Fundação de Apoio à Ciência e Tecnologia do Espírito Santo (FAPES) pelo apoio financeiro para realização deste trabalho (processo número 38939673/2007)

\section{REFERÊNCIAS BIBLIOGRÁFICAS}

AMORIM, R.C.F.; RIBEIRO, A.; LEITE, C.C.; LEAL, B.G.; SILVA, J.B.G. Avaliação do desempenho de dois métodos de espacialização da precipitação pluvial para o Estado de Alagoas. Acta Scientiarum Technology, v.30, n.1, p.87-91, 2008.

ARAÚJO, L.E.; SOUSA, F.A.S.; RIBEIRO, M.A.F.M.; SANTOS, A.S.; MEDEIROS, P.C. Análise estatística de chuvas intensas na bacia hidrográfica do rio Paraíba. Revista Brasileira de Meteorologia, São José dos Campos v.23, n.2, p.162-169, 2008.

CECÍLIO, R.A. \& PRUSKI, F.F. Interpolação dos parâmetros da equação de chuvas intensas com uso do inverso de potências da distância. Revista Brasileira de Engenharia Agrícola e Ambiental, Campina Grande, v.7, n.3, p.501-504, 2003.

FREITAS, A.J.; SILVA, D.D.; PRUSKI, F.F.; PINTO, F.A.; PEREIRA, S.B.; GOMES FILHO, R.R.; TEIXEIRA, A.F.; BAENA, L.G.N.; MELLO,
L.T.A. \& NOVAES, L.F. Equações de chuvas intensas no Estado de Minas Gerais. Belo Horizonte: Companhia de Saneamento de Minas Gerais; Viçosa: Universidade Federal de Viçosa, 2001. 65p.

MELLO, C.R.; LIMA, J.M.; SILVA, A.M.; MELLO, J.M. \& OLIVEIRA, M.S. Krigagem e inverso do quadrado da distância para interpolação dos parâmetros da equação de chuvas intensas. Revista Brasileira de Ciências do Solo, Viçosa, v.27, n.5, p.925-933, 2003.

MYERS, J.C. Geostatistical error management: quantifying uncertainty for environmental sampling and mapping. New York: Van Nostrand Reinhold, 1997. 571p.

OLIVEIRA, L.F.C.; CORTÊS, F.C.; BARBOSA, F.O.A.; ROMÃO, P.A.; CARVALHO, D.F. Estimativa das equações de chuvas intensas para algumas localidades no Estado de Goiás pelo método da desagregação de chuvas. Pesquisa Agropecuária Tropical, Goiânia, v.30, n.1, p.23-27, 2000.

PINTO, F. R. L. Equação de intensidade-duraçãofrequencia para os Estados do Rio de Janeiro e Espírito Santo: estimativa e espacialização. Viçosa MG: UFV, 1999. 70 f. Dissertação (Mestrado em Engenharia Agrícola) - Universidade Federal de Viçosa, Viçosa, MG, 1999.

PRUSKI, F.F.; CALIJURI, M.L.; BHERING, E.M.; SILVA, J.M.A. Metodologia baseada no uso de sistemas de informações geográficas para obtenção de equações de chuvas intensas em qualquer localidade do Estado do Paraná. Engenharia na Agricultura, Viçosa, v.5, n.3, p.254-265, 1997.

PRUSKI, F.F.; TEIXEIRA,A.F.; SILVA, D.D.; CECÍLIO, R.A.; SILVA, J.M.A. Plúvio 2.1: Chuvas intensas para o Brasil. In: PRUSKI, F.F.; SILVA, D.D.; TEIXEIRA, A.F.; CECÍLIO, R.A.; SILVA, J.M.A.; GRIEBELER, N.P. (Org.) HIDROS: dimensionamento de sistemas hidroagrícolas. 1 ed. Viçosa: Editora UFV, 2006, p.15-25.

SILVA, D.D.; GOMES FILHO, R.R.; PRUSKI, F.F.; PEREIRA, S.B. \& NOVAES, L.F. Chuvas intensas no Estado da Bahia. Revista Brasileira de Engenharia Agrícola e Ambiental, Campina Grande, v.6, n.2, p.362367, 2002. 
SILVA, D.D.; PEREIRA, S.B.; PRUSKI, F.F.; GOMES FILHO, R.R.; LANA, A.M.Q. \& BAENA, L.G.N. Equações de intensidadeduração-freqüência da precipitação pluvial para o Estado de Tocantins. Engenharia na Agricultura, Viçosa, v.11, n.1-4, p. 7-14, 2003.

SILVA, D.D.; PINTO, F.R.L.; PRUSKI, F.F. \& PINTO, F.A. Estimativa e espacialização dos parâmetros da equação de intensidadeduração-freqüência da precipitação para o Rio de Janeiro e o Espírito Santo. Engenharia Agrícola, Jaboticabal, v.18, n.33 (,p.22-33, 1999.

VILlELA, S.M.; MATTOS, A. Hidrologia aplicada. São Paulo: Editora McGraw-Hill do Brasil Ltda., 245p. 1975. 